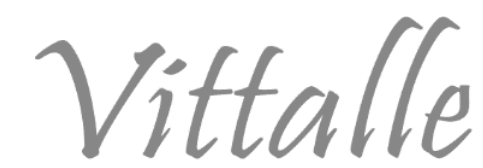

\title{
Análise temporal de descarte de bolsas de sangue por sorologia positiva no Hemonúcleo de Redenção - HENRE - (Pará) de 2010 a 2017
}

\author{
Carla Andreia Resende Rodrigues ${ }^{\mathrm{a}}$, Anna Clara Resende Martins ${ }^{\mathrm{b}}$, Diego Pereira da \\ Silva $^{c}$, Fernanda do Espirito Santo Sagica ${ }^{\mathrm{d}}$, Edivaldo Herculano Corrêa de Oliveira ${ }^{\text {a,d,* }}$

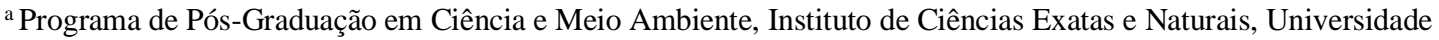 \\ Federal do Pará, Belém, PA, Brasil \\ ${ }^{\text {b }}$ Faculdade de Ensino Superior Amazônia Reunida (FESAR), Redenção, PA, Brasil \\ ${ }^{\mathrm{c}}$ Faculdade Integrada Carajás (FIC), Redenção, PA, Brasil \\ d Seção de Meio Ambiente, Instituto Evandro Chagas, Ananindeua, PA, Brasil
}

Histórico do Artigo:

Recebido em:

08/10/2020

Aceito em:

$01 / 07 / 2021$

\section{Palavras-chave:}

Desqualificação;

hemocomponentes; sorologia reagente; ciclo do sangue; segurança.

Keywords:

Disqualification; blood components; reagent serology; blood cycle; safety.

\begin{abstract}
RESUMO
A portaria $n^{\circ} 1.353 / 2011$ estabelece aos serviços hemoterápicos o alcance de altos padrões de qualidade, critérios na produção, serviços, cuidados com o indivíduo e ambiente. Baseando-se nisso, o presente estudo teve como objetivo investigar e analisar os indicadores de desqualificação das bolsas de sangue por sorologia positiva, em um hemocentro de Redenção (PA). Para isso, foi feito uma compilação de descartes por HIV, HTLV, Hepatite B e C, Doenças de Chagas, Sífilis a partir de dados obtidos através de relatórios institucionais referentes a descartes de bolsas de sangue total. Como resultado, observou-se que do total de 25.169 candidatos à doação, 1.609 bolsas coletadas foram descartadas por sorologia positiva: 1219 Hepatite B (anti-HBs), 56 Hepatite B (HBsAG), 135 Sífilis, 60 HCV, 50 Doença de Chagas, 48 HTLV e 49 HIV. O descarte das bolsas desqualificadas seguiu as normativas preconizadas pelos órgãos competentes. Dessa forma, conclui-se que os resultados observados nessa instituição atenderam a perspectiva de identificar e analisar as causas e os processos de descartes de bolsas de sangue por sorologia reativa. Espera-se a pesquisa norteie ações no controle dessas doenças, aprimore a identificação de doadores inaptos e resulte no aumento da segurança transfusional, na redução de descarte de bolsas e na atenção aos riscos sanitários e ambientais dos serviços de hemoterapia.
\end{abstract}

Temporal analysis of disposal of blood bags by positive sorology in the Redemption Hemonucleus - HENRE - (Pará) of 2010 to 2017

\begin{abstract}
Ordinance No. 1,353/2011 establishes that hemotherapy services achieve high quality standards, criteria for production, services, care for the individual and the environment. Based on this, the present study aimed to investigate and analyze the indicators of disqualification of blood bags by positive serology, in a blood center in Redenção (PA). For this, a compilation of discards by HIV, HTLV, Hepatitis B and C, Chagas diseases, Syphilis was made from data obtained through institutional reports regarding discards of whole blood bags. As a result, it was observed that out of a total of 25,169 candidates for donation, 1,609 collected bags were discarded for positive serology: 1219 Hepatitis B (anti-HBs), 56 Hepatitis B (HBsAG), 135 Syphilis, 60 HCV, 50 Chagas disease, 48 HTLV and 49 HIV. Disposal of disqualified bags followed the rules recommended by competent bodies. Thus, it is concluded that the results observed at this institution met the perspective of identifying and analyzing the causes and processes of disposing of blood bags by reactive serology. The research is expected to guide actions in the control of these diseases, improve the identification of unsuitable donors and result in increased transfusion safety, reduction of the disposal of bags and attention to the health and environmental risks of hemotherapy services.
\end{abstract}

\footnotetext{
* Autor correspondente: ehco@ufpa.br (Oliveira E. H. C.)
} 


\section{Introdução}

A transfusão de sangue e hemocomponentes é um dos relevantes avanços da terapêutica moderna (1). Assim como o sangue, a obtenção dos hemocomponentes e hemoderivados origina-se da doação, que no Brasil deve ser altruísta, voluntária, anônima e não gratificada direta ou indiretamente, em conformidade com o artigo $1^{\circ}$ da RDC - ANVISA $\mathrm{n}^{\mathrm{o}} 153$ / 2004. Os produtos gerados nos serviços de hemoterapia, a partir do sangue total, através de processos físicos são chamados de hemocomponentes. Os hemoderivados são obtidos por meio do fracionamento do plasma $(2,3)$.

Os serviços de saúde, públicos ou privados, relacionados à execução de atividades vinculadas ao ciclo produtivo de hemocomponentes e procedimentos transfusionais, têm como missão garantir que sejam alcançados altos padrões de qualidade em todas as etapas do ciclo do sangue, desde o cadastro do doador até o descarte de bolsas de sangue e hemocomponentes, estabelecendo critérios aos aspectos de produção, serviços, cuidados com o paciente e com o ambiente (4). Dessa forma, a portaria $n^{\circ} 2.712 / 2013$ estabelece a obrigatoriedade da execução de exames laboratoriais de alta sensibilidade a cada doação para detecção das seguintes infecções transmissíveis pelo sangue: sífilis; doença de Chagas; hepatite B; hepatite C; AIDS e HTLV I/II, além de malária para regiões de endemismo (5).

A hemoterapia é uma prática onerosa aos serviços públicos e aos usuários de serviços privados, pois é necessária a utilização de tecnologias modernas e de recursos humanos altamente especializados, tornando indispensável o uso racional na indicação e utilização dos hemocomponentes, considerando sempre a segurança do doador, do receptor e a disponibilidade de acesso.

Assim, é importante que os serviços de hemoterapia sigam as normas existentes, de forma clara e precisa, do ciclo do sangue, garantindo o acesso a um produto de qualidade, primordial para a saúde e vida do paciente, além de reduzir os custos envolvidos nesse processo (6). Em relação aos custos e efetividade desses serviços, a análise dos números de bolsas obtidas, processadas e descartadas, é de grande importância para o conhecimento do perfil dessas instituições e das diferenças regionais (4). Dessa forma, esse estudo tem como objetivo analisar as práticas de descarte de sangue e hemocomponentes em um hemonúcleo da Região Norte do país, a partir de dados de uma série temporal, descrevendo de forma clara e precisa os principais motivos para desqualificação das bolsas, com o intuito de auxiliar na promoção de mecanismos que contribuam para a incrementação dos processos do ciclo do sangue e na redução dos custos envolvidos

\section{Materiais e Métodos}

Esse trabalho foi aprovado pelo Comitê de Ética em Pesquisas em Seres Humanos (ICS, Universidade Federal do Pará), sob o número 3.201.818 (CAAE 03190918.8.0000.0018). Trata-se de um estudo de séries temporais com utilização de dados secundários, tendo como base procedimentos de análise documental, pesquisa bibliográfica no âmbito de saúde, social e sustentável. As informações de referência foram obtidas a partir de registros das bolsas de sangue descartadas por sorologia positiva no Centro de Hemoterapia e Hematologia do Estado do Pará - Hemonúcleo de Redenção (HENRE). Já a amostra de estudo foi o descarte dessas bolsas, no período de janeiro de 2010 a dezembro 2017.

Os dados coletados e utilizados tiveram como fonte: o sistema de dados HEMOPROD, relatório dos dados de produção, SISIND, PGRSS, POPS, planilhas mensais de coleta de resíduos, relatórios elaborados pelos setores de processamento, distribuição, enfermagem, administrativo e de qualidade vinculados ao Hemonúcleo de Redenção - Pará, no período entre janeiro de 2010 a dezembro de 2017. 
Os dados coletados foram armazenados em planilhas no Programa Microsoft Excel, com posterior cálculo das frequências, proporções, porcentagens e diferenças, que permitiram identificar os períodos de maior incidência de descartes, se foram cumpridos os protocolos de triagem, coleta, processamento, manuseio e estocagem das bolsas, a identificação das inconformidades em função dos procedimentos adotados, o mapeamento dos motivos dos descartes por sorologia positiva, a qualificação dos hemocomponentes descartados (bolsas), a quantificação dos hemocomponentes descartados (bolsas) e finalmente, a elaboração de sugestões corretivas das inconformidades identificadas.

\section{Resultados e Discussão}

De $1^{\circ}$ de janeiro de 2010 a 31 de dezembro de 2017, foram atendidos, no Hemonúcleo de Redenção, 29.953 candidatos à doação, dos quais 5.067 foram considerados inaptos no processo de triagem de doadores. Os principais motivos de inaptidão registrados durante a triagem no período analisado foram: alergias, amamentação, baixo peso, cirurgia recente, comportamento de risco, desistência, doenças cardiovasculares e dermatológicas, gripe, hanseníase, hemoglobina baixa, hipertensão, hipertireoidismo, ingestão de bebida alcoólica, lesão no local da punção, piercing, procedimento dentário, tatuagem a menos de um ano, uso de medicamentos, vacinação recente, vitiligo (doença autoimune). 25.169 candidatos foram considerados aptos e o sangue coletado encaminhado para as etapas de processamento e análise sorológicas e imunohematológicas. Em concordância com os dados nacionais, foi verificado a atenuação de doadores no HENRE, em 2017 (Figura 1).

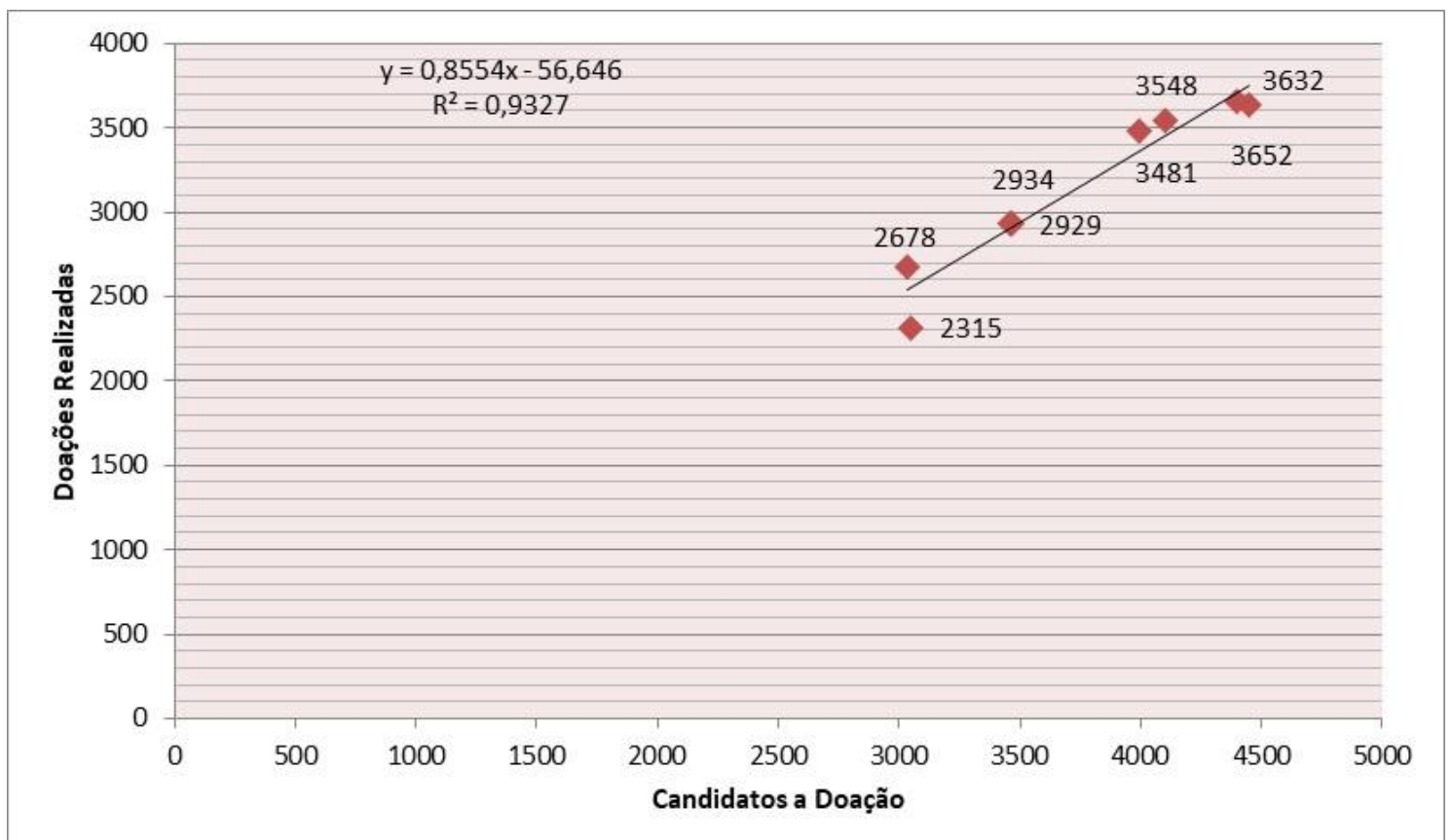

Figura 1 - Correlação entre candidatos a doação e doações realizadas, no HENRE, entre 2010 e 2017

No diagrama de dispersão tem-se o indicativo de uma forte correlação linear positiva $\left(\mathrm{r}^{2}=0,9327\right)$, entre as variáveis analisadas: candidatos à doação e doações realizadas entre 2010 e 2017 no Hemonúcleo de Redenção. O resultado apresentado demonstra estatisticamente a correlação direta de causa (candidatos a doação) e efeito (doações 
realizadas) e baixa variação entre os parâmetros estudados. A análise desse resultado reafirma o número comprovadamente baixo de candidatos a doação desistentes, assim como o reduzido número de doadores considerados inaptos através da triagem clínica, podendo ser entendido como um indicador da necessidade de aprimoramento no processo investigativo da triagem e que resultaria na redução do número de bolsas descartadas. A figura 2 retrata a correlação entre candidatos a doação e doações realizadas, no HENRE, entre 2010 e 2017.

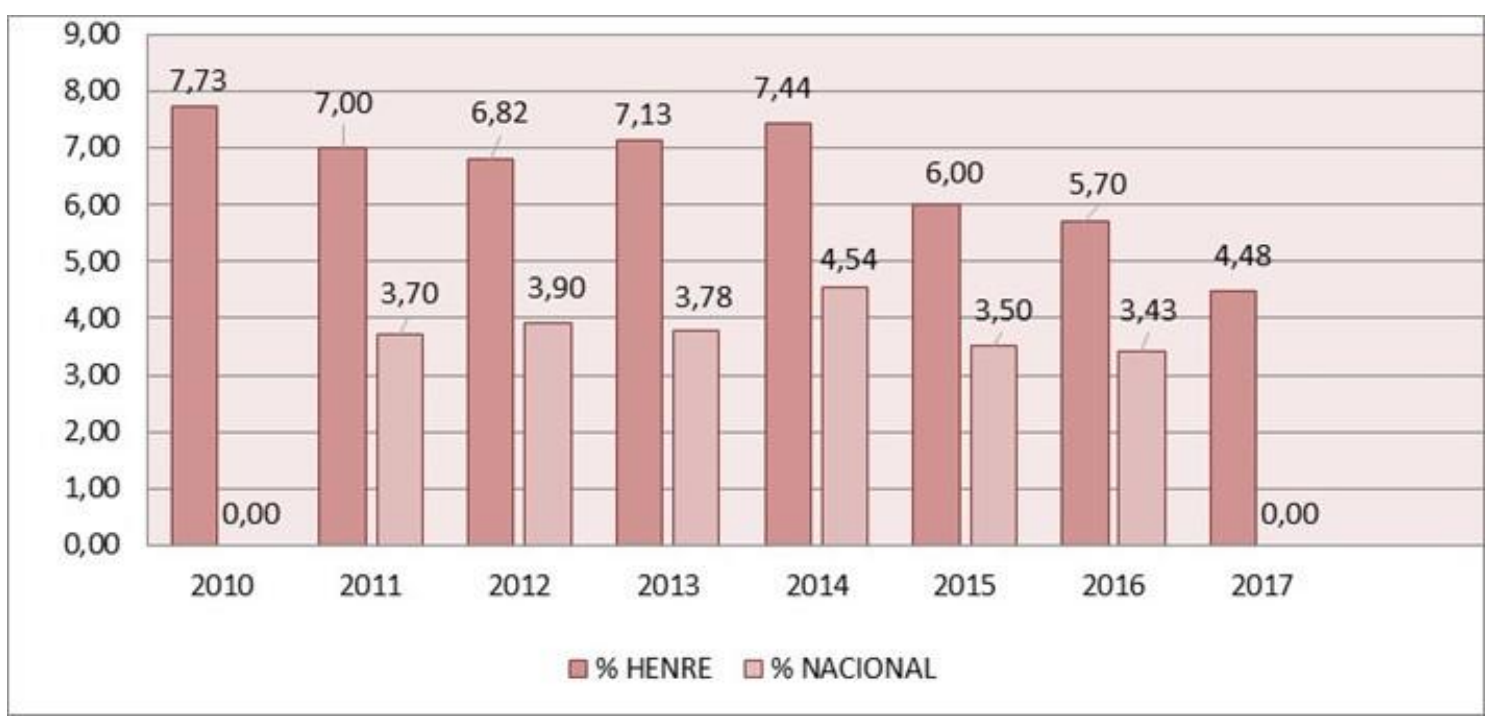

Figura 2 - Comparativo entre Inaptidão Sorológica Nacional e do HENRE, entre 2010 e 2017.

Em relação à distribuição por faixa etária dos doadores de sangue no HENRE, observouse que, na série histórica de 2010 e 2017, a predominância de doadores entre a faixa etária de 16-29 anos e do sexo masculino. Foi utilizado como instrumento de pesquisa, o Hemoprod e os dados de produção do período em estudo, disponibilizado pelo Hemonúcleo de Redenção. As ferramentas utilizadas ainda não permitem consolidar o total de candidatos à doação da faixa etária de 16 a 17 anos (menor idade) ou outra faixa etária fora dos intervalos entre 16 a 29 anos e de 30 a 69 anos, pois seguem os prérequisitos determinados pelo Ministério da Saúde. Esses resultados estão compatíveis com os dados nacionais publicados pela ANVISA em 2017.

O resultado da pesquisa também comprova a participação efetiva de doadores em idade estudantil, englobando assim alunos do ensino médio e universitários. Quanto ao comparativo entre a inaptidão sorológica nacional e os dados percentuais do HENRE entre 2010 e 2017, considerando pelo menos um marcador positivo, tem-se uma média de inaptidão sorológica de 7,47 para o HENRE e de 3,26 para o Nacional (Figura 3). 


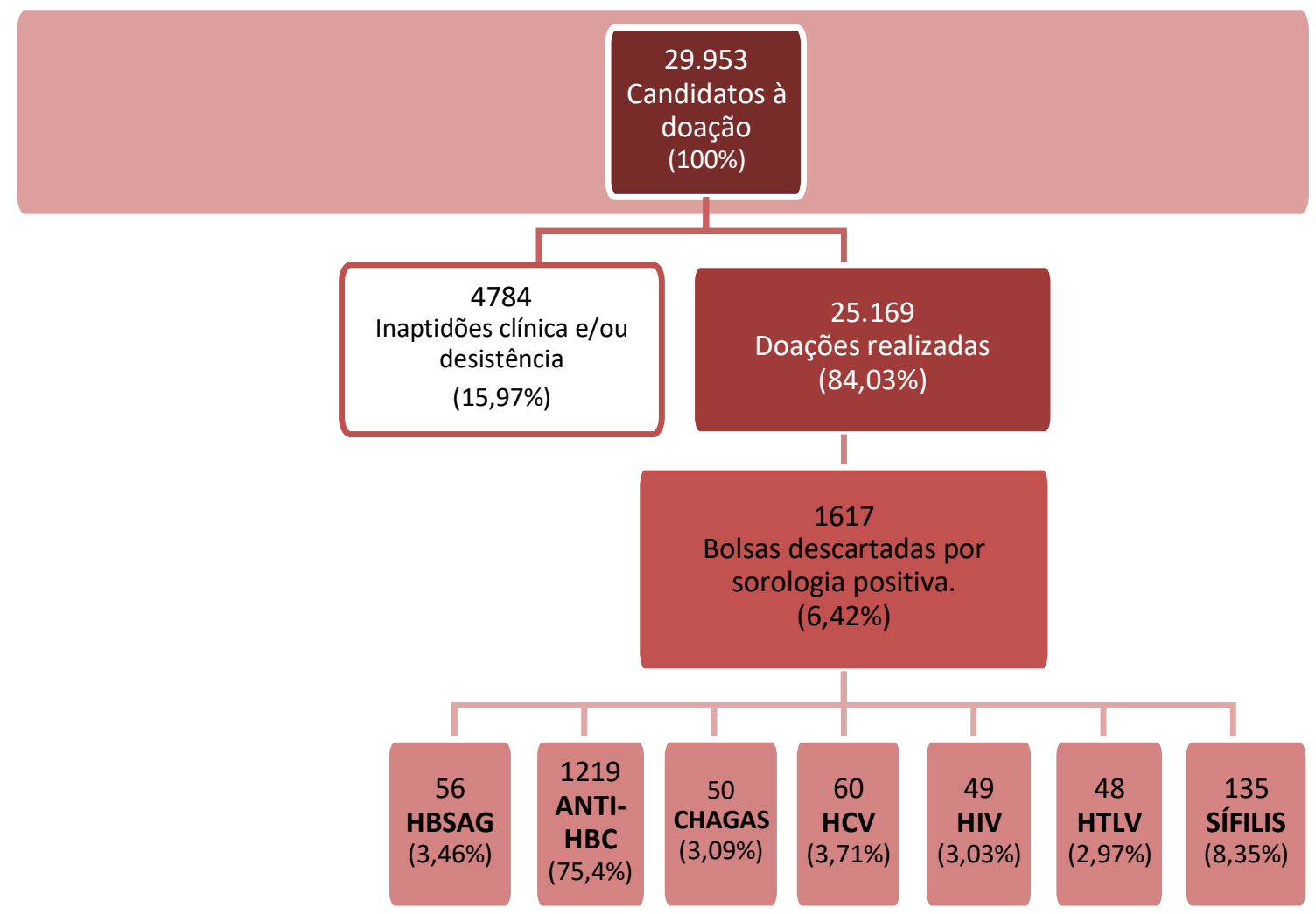

Figura 3 - Fluxograma com resumo do total de candidatos a doação de sangue e a ocorrência de descarte por sorologia positiva entre 2010 e 2017, no HENRE.

A incidência de hepatite B (anti-HBC e HBsAG), sífilis, HBC e HTLV na região atendida pelo HENRE é considerada determinante na diferença com relação aos dados nacionais apresentados (Figura 4). Em concordância, observamos nos resultados obtidos que 1.617 bolsas de sangue coletadas foram desqualificadas por sorologia positiva, sendo: 50 por doença de chagas, 1219 por hepatite B (Anti-HBc), 56 por hepatite B (HBsAg), 60 por HCV, 49 por HIV I e II, 48 por HTLV I e II, 135 por sífilis. Vale ressaltar que do total de bolsas desqualificadas, 1.219 foram assim classificadas atendendo a prerrogativa determinada pelo Ministério da Saúde quanto aos procedimentos que primam pela segurança transfusional uma vez que o resultado da sorologia determinou a imunidade do doador ou o contato com o vírus da hepatite B e não deve ser entendido como portadores da patologia em questão. 


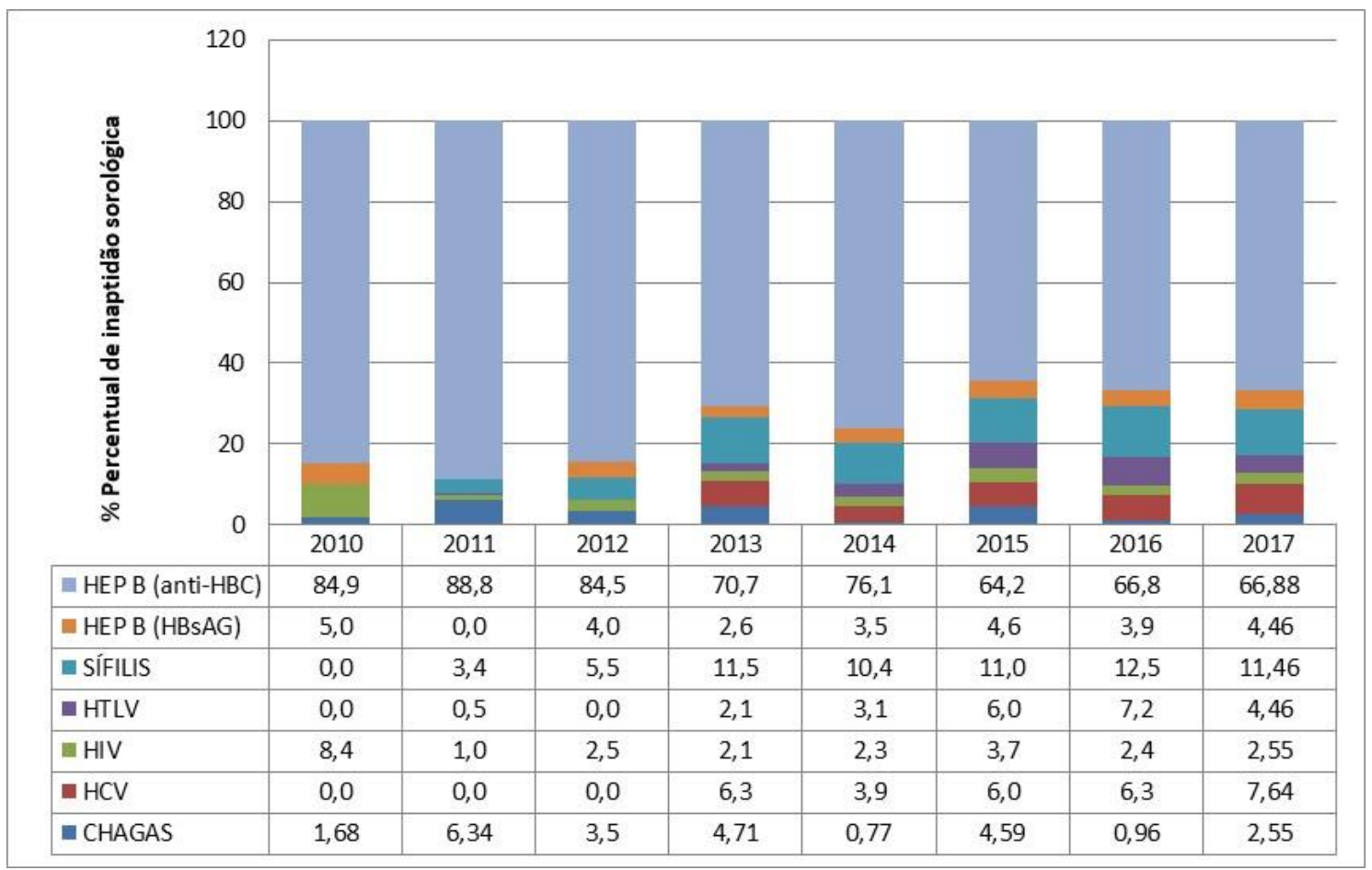

Figura 4 - Distribuição percentual da inaptidão sorológica para marcadores de doenças transmissíveis por sangue testado, no HENRE, entre 2010 e 2017.

O fluxograma com resumo do total de candidatos a doação de sangue e a ocorrência de descarte por sorologia positiva entre 2010 e 2017, no HENRE, demonstra a concordância com os dados obtidos pelo ministério da saúde e publicados no $6^{\circ}$ Boletim de Produção hemoterápica 2018. No referido boletim ficou demonstrado as maiores prevalências do marcador Anti-HBc, de Sífilis na região norte e ficando comprovado nos dados obtidos no HENRE.

Diante do fluxograma exposto é importante reforçar que existem outros motivos diversos, não incluídos no presente estudo, que geram descartes de bolsas como, por exemplo: descarte por rompimento, por caducidade, plasma feminino, por hemólise, entre outros.

Vale ressaltar que, as bolsas ficam armazenadas em refrigeradores identificados e de uso específicos até que sejam liberados os resultados das amostras de sangue colhidas durante o processamento dessas bolsas. Conforme a liberação dos resultados as bolsas de sangue serão encaminhadas para o setor de distribuição (bolsas liberadas) ou serão segregadas para o abrigo de resíduos (bolsas não liberadas).

A figura 4 descreve o percentual de descartes anual por sorologia positiva no período de 2010 a 2017 no Hemonúcleo de Redenção - Pará. Através dos dados obtidos foi possível verificar uma diminuição percentual significativa de descarte nesse período. Essa redução pode ser explicada através do aprimoramento e atenção voltada para o processo de triagem possibilitando identificar possíveis riscos de contaminação que acometeria em maior número de bolsas rejeitas e que levaria a um maior descarte e maior sobre carga de resíduos de saúde ao meio ambiente. A efetividade do processo de triagem influencia diretamente na qualidade e segurança do sangue doado.

\section{Conclusões}

A quantidade de bolsas de sangue descartadas no hemocentro analisado apresentou uma média de 6,42\% de janeiro de 2010 a dezembro 2017, sendo que a principal causa para a 
inaptidão foi a contaminação por doenças transmissíveis, destacando-se em primeiro lugar a hepatite B, seguida de sífilis, HCV, doença de Chagas, HIV I e II, e HTLV I e II. $\mathrm{O}$ ano de 2010 foi identificado como o período com maior incidência de descartes, chegando a 7,73\% do total de bolsas com sorologia positiva naquele ano.

\section{Agradecimentos}

Os autores gostariam de agradecer ao apoio recebido da Fundação HEMOPA, em especial a Silvia Cristina de Oliveira (HENRE) e Kati Seixas (GELES).

\section{Conflito de Interesse}

Os autores declaram que não há conflitos de interesse.

\section{Referências}

1. Ministério da Saúde. Guia para o uso de hemocomponentes. Brasília: Editora do Ministério da Saúde, 2009.

2. BRASIL. $1^{\circ}$ Boletim Anual de Produção Hemoterápica. Agência Nacional de Vigilância Sanitária, 2011.

3. Silva KFN, Soares S, Iwamoto HHA. Prática transfusional e a formação dos profissionais de saúde. Rev. Bras. Hematol. Hemoter. 2009; 31(6).

4. Reis DJC, Aleluia IRS, Martins MMF, Pinto Junior EP. Análise da distribuição de hemocomponentes na hemorrede do Distrito Federal. Arq. Cienc. Saúde UNIPAR. 2017; 21(2): 93-98.

5. BRASIL, Ministério da Saúde - RDC/ANVISA No 2.712. Determina o Regulamento Técnico para os procedimentos hemoterápicos. Brasília, 2013.

6. Covo MZ, Cruz EDA, Mauricio AB, Batista J, Souza LALS. Financial costs of whole blood and blood component disposals in a Brazilian coordinating blood center. Rev. Gaúcha Enferm. 2019; 40: e20190033. 